\title{
Médicos reservistas en el Líbano o el cierre de la profesionalización de las Fuerzas Armadas españolas
}

\author{
Quesada González JM. ${ }^{1}$
}

Sanid. mil. 2013; 69 (3): 215-220; ISSN: 1887-8571

\begin{abstract}
RESUMEN
Han existido oficiales médicos reservistas desde las primeras décadas del siglo XX. Primero encuadrados en la Escala de Complemento y ahora formando parte de la Reserva Voluntaria, un número nada despreciable de españoles lleva casi un siglo estando disponible para aportar capacidades sanitarias suplementarias a las Fuerzas Armadas, del mismo modo que sucede en la gran mayoría de los países miembros de la Alianza Atlántica. La disponibilidad es aún más real actualmente, dado que todos ellos están en dicha situación por propia voluntad, manifestando de esa manera su derecho y su deber de defender a España. Desde 2007, los oficiales reservistas voluntarios del Cuerpo Militar de Sanidad han estado acudiendo a la Unidad Militar de Emergencias, entre otras, para prestar sus servicios, pero no ha sido hasta 2012 que una de ellos ha salido fuera de las fronteras españolas, pudiendo afirmar ahora que el modelo profesional de Fuerzas Armadas se encuentra totalmente implantado.
\end{abstract}

PALABRAS CLAVE: Reserva, Reservista, Sanidad Militar, Capacidad, Internacional.

Medical Reservists in Lebanon or closing of the professionalization process in the Spanish Armed Forces.

SUMMARY: Since the early decades of the twentieth century, medical reserve officers have been part of the national defence. Initially as members of the «Escala de Complemento» and nowadays as voluntary reservists, quite a number of Spaniards have been available for almost a century to provide additional medical capabilities to the military, as it is usual in most NATO members. Availability is even realler today, since all of them belong to there serve by their own choice, thereby expressing their right and duty to defend Spain. From 2007 on, Medical Service Corps reserve officers were assigned, among others corps, to the Military Emergency Unit to fulfill their duties, but not till 2012 one of them was deployed abroad. It may be said that this assignment fully implemented the professional military model.

KEY WORDS: Reserve, Reservist, Medical Service, Capacity, International.

\section{INTRODUCCIÓN}

El pasado mes de febrero, volvió a su trabajo habitual en el Servicio Aragonés de Salud de Teruel el primer reservista voluntario español desplegado en una misión internacional. Se trata de la Teniente Médico RV Jacqueline Vázquez Rodríguez, quien durante dos meses había formado parte del escalón sanitario de la Agrupación Libre Hidalgo VIII, destacado en el Líbano.

Este hecho que, a primera vista parece poco relevante, adquiere toda su importancia cuando se toma en consideración que la Reserva Voluntaria lleva casi diez años en situación de aportar capacidades suplementarias a las Fuerzas Armadas. La componen más de cinco mil ciudadanos españoles maduros y sólidamente preparados que han decidido vincularse voluntaria y temporalmente a la Defensa Nacional y que, a pesar de ello, no habían sido desplegados hasta este momento en una zona donde estuviesen operando Unidades Armadas.

Por otra parte, transcurridas casi dos décadas desde que la Ley del Servicio Militar de 1991 permitiera por primera vez nutrir significativamente las Unidades con soldados profesionales,

'Tte. Reservista Voluntario.

Director de Operaciones de Schindler, S.A.

Ingeniero Industrial. Doctor en Paz y Seguridad Internacional.

Dirección para correspondencia: jose_miguel_quesada@hotmail.com

Recibido: 11 de marzo de 2013

Aceptado: 30 de abril de 2013 ha quedado de esta manera cerrado el ciclo de la profesionalización de las Fuerzas Armadas dado que los reservistas ya han comenzado a compartir todo tipo de misiones con las fuerzas regulares. Durante estos años se ha venido concibiendo e implantando un modelo de reserva que, como no podía ser de otra manera, ha recibido influencias de los demás países de la Alianza y de los agentes involucrados en la defensa. Reservistas voluntarios - muchos de los cuales devienen luego en reservistas honoríficos-, reservistas de especial disponibilidad y reservistas obligatorios componen las fuerzas de las que España dispone en potencia y que, con sus particularidades propias, resultan homologables a las de otros aliados.

\section{LA IMPORTANCIA DE LOS MÉDICOS RESERVISTAS PARA LA OTAN}

Las Fuerzas Armadas estadounidenses terminaron la Segunda Guerra Mundial con 40.000 oficiales médicos, cuando sólo tenían mil en activo al comienzo de la misma. Asimismo, Canadá movilizó durante la contienda a 4.000 profesionales de este tipo. Estas cifras son muy representativas del potencial que supone para la Defensa Nacional disponer de una organización que mantenga a este personal en situación sedentaria, con algún tipo de encuadramiento y debidamente adiestrado ${ }^{1}$.

Ha cambiado mucho el escenario estratégico desde el final de la Segunda Guerra Mundial. Sin embargo, la OTAN es toda- 
vía consciente de que reservistas adaptados a los nuevos tiempos pueden resultar útiles, sobre todo cuando se habla de la lucha contra insurgencia, donde las operaciones se centran en la población y en las que, por tanto, el entorno humano o tecnológico es capital. Además, la Alianza piensa que es un verdadero triunfo poder disponer de especialistas que hagan de puente entre el mundo civil y el militar mediante la aportación de importantes conocimientos civiles, reforzados con algunas competencias puramente militares, siendo este principio de plena aplicación a los profesionales de la Medicina ${ }^{2}$.

Estados Unidos, que es el paradigma de empleo intensivo de la reserva militar, cuenta con un millón de reservistas de todo tipo. Desde los atentados del 11-S, 800.000 de ellos han sido desplegados fuera de sus fronteras. En cuanto a los médicos, nada menos que el 70 por ciento de las vacantes de sanidad de las plantillas orgánicas de sus agrupaciones expedicionarias son ocupadas por civiles, que han abandonado durante unos meses la consulta privada o el quirófano para ponerse el uniforme ${ }^{3}$.

\section{ANTECEDENTES HISTÓRICOS ESPAÑOLES}

Entre el último tercio del siglo XIX y los primeros años del siglo $\mathrm{xx}$, las potencias militares europeas reconocieron que necesitaban una clase de oficialidad que permitiera poner en pie de guerra, rápidamente, a grandes masas de reservistas. El elevado volumen de soldados que participaban en las contiendas debía proceder de una bien organizada reserva, pero era demasiado costoso que la mayor parte de sus mandos, los oficiales de empleos inferiores, destinados a liderar secciones y compañías -y accidentalmente batallones-, perteneciesen a las escalas profesionales ${ }^{4}$.

\section{Primera época: oficiales médicos de complemento}

En España, la primera disposición que instauró una oficialidad de estas características fue la ley de reclutamiento de 1912. Sin embargo, el nacimiento de la Escala de Complemento española suele fijarse en la fecha en que fue citada por primera vez en un texto legal, es decir, en 1918. Se trataba de oficiales que, para empezar, habían pagado una suma por realizar un servicio militar más corto y en condiciones privilegiadas. Evidentemente, se les presumía una formación académica superior a la media. Una vez que se pertenecía a este grupo de favorecidos y si manifestaban la voluntariedad de pertenecer a la citada escala, se les impartía durante un corto espacio de tiempo adicional una instrucción mínima para desempeñar sus cometidos de oficial.

En cuanto a la asignación de destinos, la intención era que los estudiantes o licenciados de determinadas carreras universitarias consideradas útiles para el Ejército fueran enviados, preferentemente, a aquellos Cuerpos o Servicios que tenían más relación, por su carácter técnico, con el mundo civil. Como es lógico, farmacéuticos, médicos y veterinarios eran preferidos en los servicios homónimos. Particularmente los segundos alcanzaban los empleos de alférez, teniente o capitán médico de complemento si, al terminar el período de instrucción, poseían el título de licenciado en Medicina; mientras que obtenían los de alférez, teniente o capitán de complemento de las tropas de sanidad si no habían llegado a acabar la carrera.

La consideración que se les tenía era tal, que el Ejército prefería ocupar las vacantes profesionales de dichos Cuerpos con médicos, farmacéuticos o veterinarios de complemento. Además, lo mismo que sus compañeros de otras especialidades fundamentales, podían concursar con prioridad a determinados puestos de la función pública con un perfil compatible «con la superior cultura y categoría militar» ${ }^{5}$.

Desafortunadamente, hasta los años cuarenta, la Escala de Complemento fue un completo fracaso porque no llegó a alcanzar un tamaño mínimamente significativo. Su principal defecto era la ausencia de incentivos para pertenecer a ella. La falta de una adecuada oficialidad movilizable repercutió en las medidas que hubo que tomar en los primeros meses de la Guerra Civil. De los 6.156 oficiales de complemento existentes en 1936, sólo se tiene constancia de que combatiera la mitad, obligando a ambos contendientes al ascenso apresurado de sus suboficiales y a la improvisación de escuelas de circunstancia para mandos ${ }^{6}$.

\section{Segunda época: modalidades universitarias de oficialidad movilizable}

La ley de reclutamiento de 1940 suprimió para siempre los privilegios que algunos mozos podían obtener a cambio del pago de una cuota. Por lo tanto, el ingreso en la Escala de Complemento hubo de ser cambiado. Durante el régimen franquista dicha Escala se nutrió con universitarios que habían sido instruidos militarmente durante sus estudios y al final de éstos, mientras cumplían la obligación de realizar el servicio militar. Dichos programas de formación sufrieron cierta evolución dependiendo de las circunstancias interiores y exteriores de España.

No obstante, durante los primeros años de vida de esta segunda época no hubo oficiales reservistas vinculados a la Sanidad Militar. Farmacéuticos, médicos y odontólogos eran enviados a Infantería, mientras que veterinarios servían en Caballería. En 1967, por primera vez, se crearon algo más de doscientas plazas de sanidad en el programa de instrucción de la Instrucción Premilitar Superior del Ejército de Tierra (tabla 1). Nótese cómo se pueden identificar en dicha tabla algunos singulares comportamientos sociales y militares, como fue el fabuloso incremento de la población universitaria que tuvo lugar a finales de los años sesenta, y en la primera mitad de los setenta, la reducción de unidades y de personal que comenzó en la segunda mitad de los ochenta y la desafección generalizada hacia el servicio militar obligatorio durante el tramo final.

Desde la posguerra y hasta la suspensión del servicio militar obligatorio, 9.011 estudiantes de carreras relacionadas con las ciencias de la salud, decidieron realizar su prestación personal de forma que permanecieran disponibles como oficiales reservistas hasta la edad de retiro de los militares profesionales, hasta cumplir quince años desde su ingreso en filas — con el máximo de treinta y cuatro años de edad-, o hasta tres años después de la finalización del servicio activo, dependiendo de la legislación que les correspondiese a cada uno. 
Tabla 1. Alféreces movilizables del Ejército de Tierra, pertenecientes a los Cuerpos de Sanidad Militar, de Farmacia Militar y de Veterinaria Militar, que terminaron su instrucción en el periodo 1967-2001. Fuente: Diario Oficial del Ministerio del Ejército (1943-1977), Diario Oficial del Ejército (1977-1985) y Boletín Oficial del Ministerio de Defensa (1985-2001).

\begin{tabular}{|c|c|c|c|c|c|c|c|c|c|}
\hline Año & 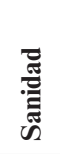 & 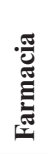 & 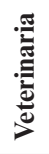 & हैं & Año & 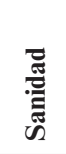 & 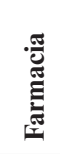 & 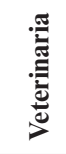 & हैँ \\
\hline 1967 & 191 & 15 & 6 & 212 & 1985 & 205 & 10 & 11 & 226 \\
\hline 1968 & 187 & 15 & 6 & 208 & 1986 & 218 & 10 & 10 & 238 \\
\hline 1969 & 192 & 16 & 6 & 214 & 1987 & 199 & 11 & 9 & 219 \\
\hline 1970 & 279 & 13 & 6 & 298 & 1988 & 190 & 8 & 10 & 208 \\
\hline 1971 & 275 & 15 & 6 & 296 & 1989 & 172 & 4 & 5 & 181 \\
\hline 1972 & 277 & 15 & 6 & 298 & 1990 & 336 & 15 & 22 & 373 \\
\hline 1973 & 519 & 18 & 16 & 553 & 1991 & 179 & 27 & 58 & 264 \\
\hline 1974 & 558 & 20 & 20 & 598 & 1992 & 198 & 34 & 41 & 273 \\
\hline 1975 & 547 & 20 & 23 & 590 & 1993 & 8 & 13 & 18 & 39 \\
\hline 1976 & 563 & 23 & 23 & 609 & 1994 & 66 & 16 & 20 & 102 \\
\hline 1977 & 345 & 12 & 12 & 369 & 1995 & 128 & 14 & 20 & 162 \\
\hline 1978 & 357 & 12 & 11 & 380 & 1996 & 93 & 17 & 24 & 134 \\
\hline 1979 & 426 & 12 & 12 & 450 & 1997 & 74 & 17 & 22 & 113 \\
\hline 1980 & 244 & 10 & 8 & 262 & 1998 & 66 & 18 & 21 & 105 \\
\hline 1981 & 249 & 10 & 7 & 266 & 1999 & 24 & 11 & 15 & 50 \\
\hline 1982 & 242 & 10 & 9 & 261 & 2000 & 5 & 1 & 5 & 11 \\
\hline 1983 & 200 & 7 & 8 & 215 & 2001 & 0 & 0 & 0 & 0 \\
\hline 1984 & 215 & 10 & 9 & 234 & & & & & \\
\hline \multicolumn{6}{|c|}{ TOTAL } & 8027 & 479 & 505 & 9011 \\
\hline
\end{tabular}

\section{EL MODELO ESPAÑOL DE RESERVA}

La OTAN otorga a cada país miembro un amplio margen para la organización de la reserva militar permitiendo así que puedan pertenecer a la Alianza países tan dispares, desde el punto de vista de la organización de su defensa, como Turquía o como Estados Unidos. No obstante, establece claramente que se tiene que contar con algún sistema que, ante una crisis, permita incrementar tanto los efectivos como las capacidades militares.

Una vez decidida la suspensión de la prestación personal obligatoria, quedaba agotada al mismo tiempo la histórica reserva de masas que había estado tradicionalmente ligada a la conscripción, como las dos caras que pertenecen a una misma moneda. Había que decidir qué tipo de reserva tener, elegir entre el paradigma anglosajón-cuyo elemento principal son las unidades específicas de reservistas que pueden asumir las mismas misiones que las profesionales - o el modelo continental - basado en que las Fuerzas Armadas reciben capacidades suple- mentarias del ámbito civil—, teniendo siempre presente que el voluntariado debía jugar un papel fundamental.

Finalmente, se decidió que el concepto español de reserva debía responder fielmente al paradigma continental, guardando una especial similitud con la configuración francesa. De esta manera, el gran núcleo de la reserva española está actualmente constituido por personal voluntario que permanece en situación de disponibilidad para integrarse, si fuera necesario, en las unidades existentes. La aportación suplementaria de capacidades que se espera de ellos es, principalmente, de carácter civil. Además, el modelo se completa con los reservistas de especial disponibilidad y los obligatorios(ver tabla n. ${ }^{\circ} 2$ ). En la legislación se menciona también la figura del reservista honorífico sin que por ello forme parte del concepto de reserva, dado que no se le atribuye función alguna. Contrariamente a lo que le sucede al miembro de la Réserve Citoyenne francesa, este reservista adquiere su condición en reconocimiento a los servicios prestados con anterioridad.

En 2012, se disponía de 5.417 reservistas voluntarios pertenecientes, en su mayoría, al Ejército de Tierra. Los de especial disponibilidad, ligados al modelo de reclutamiento de personal de tropa y de complemento tenían una presencia meramente testimonial - 55 personas - En cuanto a los honoríficos, 1.441 españoles habían recibido esa distinción, ya fuera por haber sido reservistas voluntarios y haber finalizado su compromiso, ya fuera por ser veteranos del servicio militar obligatorio. Por último, los hombres y mujeres que podrían llegar a ser reservistas obligatorios eran los casi tres millones de ciudadanos españoles que, a finales de 2011, tenían entre diecinueve y veinticinco años.

Actualmente, casi la mitad de los reservistas voluntarios son oficiales, mientras que un tercio son suboficiales. De esta manera, la presencia de soldados y marineros queda reducida a algo más de una quinta parte. Sin embargo, dadas las principales misiones en las que este personal puede ser de utilidad, no parece que esta desproporción entre clases sea en sí misma un problema. Aunque mayoritariamente masculina, se puede asegurar que hay más mujeres que se han sentido atraídas por esta modalidad de vinculación a la defensa, que por hacer carrera como militares profesionales.

El proceso de aportación gradual de fuerzas de reserva se encuentra en la figura 1. En situación de normalidad, los reservistas voluntarios son activados de acuerdo con los planes anuales que establecen distintos tipos de instrucción. No hace falta que suceda nada excepcional para que este colectivo contribuya, en tiempo de paz, a mejorar las capacidades de las unidades a las que están adscritos, ya sea dentro o fuera del territorio nacional.

Tan pronto se produjera un riesgo alarmante para la seguridad nacional, y las fuerzas regulares necesitaran incrementar sus capacidades, el Consejo de Ministros podría adoptar las medidas necesarias para incorporar, en primera instancia, a los reservistas voluntarios y de especial disponibilidad que se requiriesen.

Tabla 2. Clasificación de los reservistas españoles ${ }^{15}$.

\begin{tabular}{|c|c|c|c|c|}
\hline Tipo & Condición & Incorporación & Permanencia & Requisitos \\
\hline Voluntario & Voluntaria & Voluntaria, normalidad & Voluntaria & Todos los españoles, hasta los 58-61 años \\
\hline De especial disponibilidad & Voluntaria & Obligatoria, crisis & Voluntaria & Ex oficiales de complemento y tropa/marinería \\
\hline Obligatorio & Obligatoria & Obligatoria, crisis & Obligatoria & Españoles entre 19 y 25 años \\
\hline
\end{tabular}




\begin{tabular}{|c|c|c|c|}
\hline Personal & Normalidad & Escalada & $\Rightarrow \begin{array}{c}\text { Necesidades } \\
\text { excepcionales }\end{array}$ \\
\hline Reservistas voluntarios & Activación & $\begin{array}{c}\text { Incremento del } \\
\text { personal activado }\end{array}$ & \\
\hline $\begin{array}{l}\text { Reservistas de especial } \\
\text { disponiblidad }\end{array}$ & & Incorporación & \\
\hline Reservistas obligatorios & & & $\begin{array}{l}\text { Declaración general } \\
\text { de reservistas }\end{array}$ \\
\hline $\begin{array}{l}\text { Oficiales y suboficiales en } \\
\text { situación administrativa de } \\
\text { reserva }\end{array}$ & & & Movilización \\
\hline
\end{tabular}

Figura 1. Proceso de aportación gradual de reservistas16.

En el caso de los primeros, sería necesario que se les impartiera una formación específica - aparte de la formación continuada a la que normalmente están sometidos - que les permitiera adaptarse a la plaza a la que se debieran incorporar.

Posteriormente, si la escalada deviniera en crisis, el Gobierno solicitaría al Congreso autorización para emitir la declaración general de reservistas obligatorios, a quienes se les reconocería el derecho a la objeción de conciencia. En este caso, los objetores se asignarían a un destino que no requiriese el empleo de armas. Al realizar la citada declaración podrían ser movilizados también los cuadros de mando profesionales que se encontrasen en situación de reserva, potenciales jefes de las unidades de entidad entre pelotón y batallón que se constituirían con los reservistas obligatorios puestos sobre las armas.

\section{Los reservistas del Cuerpo Militar de Sanidad}

Los Cuerpos Comunes que actualmente cuentan con reservistas son el de Sanidad y el Jurídico, si bien es meramente testimonial la presencia en el segundo de ellos por lo que, en adelante, se identificarán los datos del Cuerpo Militar de Sanidad con los de la totalidad. Hecha esta salvedad, en 2012 estaban adscritos a la Sanidad Militar 561 reservistas voluntarios y tres de especial disponibilidad — ex militares de complemento-. Eso supone alrededor del diez por ciento

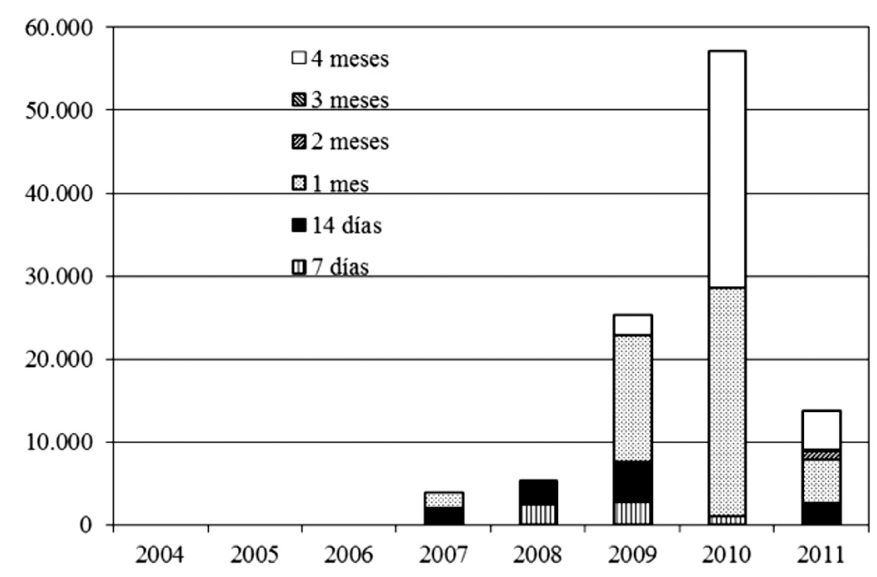

Figura 2. Días anuales de activación de los reservistas voluntarios del Cuerpo Militar de Sanidad (2004-2011) según la duración de la misma. Fuente: Ministerio de Defensa. de todo el personal reservista disponible, estando en manos de mujeres un tercio de dicha capacidad sanitaria suplementaria.

Cada año, el Consejo de Ministros establece las plazas de reservistas voluntarios que se desea cubrir, desglosadas las especialidades de Enfermería, Farmacia, Medicina, Odontología, Psicología y Veterinaria. Mediante concurso de méritos, pueden ser cubiertas por cualquier español que cumpla tanto las condiciones académicas propias del Cuerpo, como las de acceso que dependen, a su vez, de la procedencia de los candidatos, ya sean civiles, militares de complemento que hayan finalizado su compromiso o militares de carrera que hayan renunciado a su condición castrense. Una vez finalizado el período de formación, los aspirantes adquieren el empleo de alférez o cualquier otro superior que hubiesen tenido en su anterior vinculación con las Fuerzas Armadas. A partir de ahí se puede ascender cumpliendo determinadas condiciones, hasta capitán. En 2012, la distribución por empleos era de un comandante, cuatro capitanes, 84 tenientes y 428 alféreces del Cuerpo Militar de Sanidad.

En cuanto a su preparación laboral, es preciso decir que el 86 por ciento de los reservistas voluntarios del Cuerpo Militar de Sanidad supera los cuarenta años de edad y que el 48 por ciento están en la cincuentena. Dada la veteranía del colectivo, es de suponer que puedan aportan lo que mejor saben hacer en el ejercicio diario de su profesión, que debe de ser mucho.

Si se repara en el número de efectivos existente, se comprobará que los reservistas voluntarios de Sanidad podrían cubrir más de la cuarta parte de todas las vacantes del Cuerpo. Si se excluyen las que requieren empleos no alcanzables por ellos, los efectivos en reserva son la mitad. Y esta proporción sería aun más grande si se descontaran las vacantes no cubiertas por falta de candidatos. No cabe duda de que, inmersos en una notoria escasez de médicos militares, los reservistas sanitarios constituyen una capacidad adicional nada despreciable? .

\section{ACTIVACIONES DE LOS OFICIALES RESERVISTAS DE SANIDAD}

Una vez adquirida su condición, este personal puede ser llamado para realizar actividades de formación continuada o para prestar servicio en unidad, ya sea en territorio nacional o en misiones internacionales, requiriéndose su autorización expresa para poder ser asignado a estas últimas. La formación continuada se justifica porque su capacidad militar no sólo debe ser adquirida, sino que también hay que conservarla. Para ello, el subsecretario de Defensa debe aprobar planes anuales que contemplen «ejercicios de instrucción y adiestramiento, cursos y seminarios de perfeccionamiento, y prácticas de adaptación a la plaza asignada». La asistencia a estas actividades formativas es retribuida.

Algunos oficiales generales que han ocupado cargos de responsabilidad en el Ministerio de Defensa piensan que este personal es necesario para afrontar los retos estratégicos de la sociedad actual. Es el caso del general Echepare, uno de los primeros jefes de la Oficina General de Reservistas. Por su parte, el almirante Cayetano - ex subdirector general de Reclutamiento- dejó escrito en 2011 que la variedad y grado de especialización de este 


\section{Médicos reservistas en el Líbano o el cierre de la profesionalización de las Fuerzas Armadas españolas}

personal no se encuentra entre los componentes de las Fuerzas Armadas y que, por eso, habría que reclamar su participación «activa y eficaz en la seguridad y la defensa», considerando que su incorporación podría ser «apropiada» ${ }^{8}$.

$\mathrm{Y}$ tiene sentido contar con recursos conectados con el mundo civil, dado que las misiones más probables para las Fuerzas Armadas durante el primer tercio del siglo XXI serán las de estabilización, con lo que «crecerá la demanda de reservistas voluntarios que acrediten capacidades apropiadas para el desarrollo de las tareas concretas que requieren este tipo de misiones». Dichas misiones serán las que contribuyan a crear un ambiente seguro, proporcionar a la población los servicios fundamentales, apoyar el desarrollo económico y social, construir —o reconstruir, en su caso- las infraestructuras más urgentes y proporcionar ayuda humanitaria9.

\section{En territorio nacional}

En este sentido, médicos y enfermeros son los que tienen mayor probabilidad de ser activados para prestar servicio en Unidades, Centros u Organismos de la Defensa, dadas las carencias existentes en estas especialidades. Y el destino en el que se les emplea usual y preferentemente es la Unidad Militar de Emergencias, donde prestan servicio en número muy importante desde 2007. Allí facilitan y complementan la labor del personal sanitario de plantilla con resultado muy satisfactorio, según la valoración realizada por el Ministerio de Defensa ${ }^{10}$.

En la figura $\mathrm{n}^{\circ} 2$ se encuentra la evolución anual de los días de activación de los reservistas del Cuerpo Militar de Sanidad en el tramo 2004-2011 según la duración, que puede oscilar entre la semana de formación continuada y los cuatro meses de prestación de servicio en unidad. Se advierte una tendencia creciente hasta 2010, el año de mayor empleo de este recurso, con 57.116 días en total. A partir de entonces es muy evidente una bajada en las activaciones a valores anteriores a 2009 debido, fundamentalmente, a razones económicas.

\section{Fuera de las fronteras españolas}

En cuanto a las activaciones para unirse a misiones en el extranjero, la primera oportunidad surgió en 2005, cuando dos médicos y tres enfermeros - alféreces reservistas voluntariospermanecieron en Sumatra (Indonesia) durante dos meses, formando parte de la Operación Respuesta Solidaria. Allí colaboraron en las labores de rescate y reconstrucción que la Comunidad Internacional puso en marcha tras el maremoto que asoló la isla ${ }^{11}$.

A pesar de que no consta que la experiencia fuera negativa, la posibilidad de utilizar este recurso en Misiones de Paz fue ignorada durante el resto de esa legislatura y la siguiente, desoyendo las reclamaciones - de algún Estado Mayor y de varias Direcciones Generales del Ministerio - en el sentido de emplear médicos y enfermeros reservistas para aliviar la presión a la que se estaba sometiendo a los escasos efectivos profesionales. Como dijo el general Echepare en 2009, «la voluntariedad de participación de los reservistas, en cualquier misión, está fuera de toda duda.
Ya lo han demostrado en la Unidad Militar de Emergencias a lo largo de los tres últimos años y se encuentran a la espera deuna oportunidad para hacerlo en el exterior.Se la han ganado» ${ }^{12}$. Hay otras personas involucradas en la defensa que piensan que habría que emplear con mayor profusión a los reservistas voluntarios como, por ejemplo, la diputada Irene Lozano - del partido Unión, Progreso y Democracia (UPyD)_, que es miembro de la comisión de Defensa del Congreso. Respondiendo a una pregunta sobre la sostenibilidad económica de las Fuerzas Armadas actuales, ella dijo que «como propuesta o idea a desarrollar, considerando la muy probable reducción de efectivos, está la mayor implicación de los Reservistas Voluntarios» ${ }^{13}$.

Hubo que esperar hasta bien avanzado el año 2012 para que el ministro de Defensa autorizase, por primera vez en la historia española, que reservistas voluntarios participasen en misiones de mantenimiento de la paz. Aunque la orden ministerial permitía que los designados fueran enviados tanto a la operación Libre Hidalgo como a la Atalanta, sólo se seleccionó finalmente a una alférez médico - especialista en medicina familiar y comunitaria - para prestar asistencia sanitaria al grupo táctico de la primera de ellas, quien permaneció un tiempo aproximado de dos meses en la base Miguel de Cervantes de Marjayún.

Cuando esta activación ya se encontraba en curso, en diciembre de 2012, sucedió que la Unidad Militar de Emergencias acreditó ante la entidad específica de la ONU que su equipo de búsqueda y rescate urbano (UrbanSearchAnd Rescue, USAR) - creado, como en numerosos países, para auxiliar de manera rápida y eficaz a una nación azotada por un desastre - cumplía con los requisitos necesarios para intervenir en este tipo de situaciones. Sin embargo, las carencias de personal sanitario que esta unidad tenía en su actividad en territorio nacional habrían podido poner en riesgo el éxito de una eventual misión en suelo extranjero. Por ello, de inmediato, el Ministerio de Defensa extendió a los equipos USAR la posibilidad de activar a reservistas fuera de las fronteras españolas ${ }^{14}$.

No se podía dar por cerrada la profesionalización de las Fuerzas Armadas hasta que los reservistas no participasen en las mismas misiones que sus compañeros regulares. La necesidad de involucrar recursos provenientes del ámbito civil para hacer frente a los retos estratégicos actuales es evidente. Así lo ha establecido la OTAN y así lo están haciendo la mayoría de sus miembros, particularmente los más comprometidos con los objetivos comunes de seguridad. Se cuenta con recursos capacitados que se han acercado voluntariamente a las Fuerzas Armadas y que, posiblemente, requieren de alguna instrucción militar adicional. Ha existido la voluntad política de que una de ellos participe en una misión internacional. El camino está abierto y sólo queda recorrerlo.

\section{BIBLIOGRAFÍA}

1. Confederación Interaliada de Oficiales Médicos de Reserva, Actas del congreso de 20 y 21 de noviembre de 1948. Disponible en: http://www.ciomr. org/download/CIOMR_Minutes_20-21Nov48_lowres.pdf (consultada por última vez el 6 de marzo de 2013).

2. Lasconjarias G. L'OTAN et le futur des réserves. Revue Armée et Défense: Unión Nacional de Oficiales de Reserva y de Organizaciones de Reservistas (UNOR); 2012;2/4:19. 
3. Weitz R. The reserve policy of nations: a comparative analysis. Strategic Studies Institute; 2007:5-12.

4. Villalba J. Organización de la Educación física e Instrucción premilitar en Francia, Suecia, Alemania e Italia (Viaje de estudio): Talleres del Depósito de la Guerra, 1927:62.

5. Real orden circular organizando la oficialidad y clases de complemento de las diversas Armas y Cuerpos del Ejército y real orden circular, ampliando en la forma que se indica la base 2. ${ }^{\text {a }}$ del real decreto-ley de 6 de septiembre de 1925 , relativo a la provisión de destinos públicos entre licenciados del Ejército de 18 de diciembre: Gaceta de Madrid, 1929;353.

6. Gárate Córdoba JM. Improvisación de mandos en 1936, I. Alféreces provisionales, Revista de Historia Militar: Instituto de Historia y Cultura Militar; 2010:53-114.

7. Real decreto $1311 / 2004$, de 28 de mayo, por el que se fijan las plantillas reglamentarias de cuadros de mando de las Fuerzas Armadas para el período 2004-2009: Boletín Oficial del Estado, 2004;141.

8. Cayetano L, op. cit.:24-26.

9. Echepare Fernández B. Los reservistas voluntarios, Revista Atenea: Editorial i2v, 2009;12:45.
10. Respuesta al diputado Ignacio Cosidó (PP) sobre medidas para paliar la falta de conocimientos de determinados colectivos de reservistas voluntarios, 11 de diciembre: Boletín Oficial de las Cortes Generales - Congreso de los Diputados, 2009;D-344: 409.

11. Resolución 431/01461/05 del subsecretario de Defensa, 25 de enero: Boletín Oficial del Ministerio de Defensa, 2005;20.

12. Echepare Fernández B, op. cit.: 46.

13. Lacave L, Lozano I. «UPyD pide una profunda revisión de la Ley de la Carrera Militar», Ateneadigital.es, Disponible en: http://www.revistatenea.es/REVISTAATENEA/REVISTA/ARTICULOS/GESTIONNOTICIAS_7591_ESP.ASP (consultada por última vez el 10 de marzo de 2013).

14. Orden ministerial 95/2012 que modifica la orden ministerial66/2012, de 10 de septiembre, por la que se autoriza la incorporación de reservistas voluntarios para participar en determinadas misiones de las Fuerzas Armadas en el extranjero, 21 de diciembre: Boletín Oficial del Ministerio de Defensa, 2012; 8.

15. Cayetano L. Los reservistas voluntarios: retos y consolidación del modelo, Boletín de información del CESEDEN: Ministerio de Defensa, 2012;321:15.

16. Aguirre Scandella J. Presente y futuro de la Reserva Voluntaria en las Fuerzas Armadas españolas, Revista Ejército: Ministerio de Defensa, 2009;816:76. 